\title{
Estado de conservación de los vertebrados terrestres de Oaxaca, México
}

\section{Conservation status of the terrestrial vertebrates from state of Oaxaca, Mexico}

\author{
Mario C. Lavariega ${ }^{1 *}$, Natalia Martín-Regalado ${ }^{1}$, Alina Gabriela Monroy-Gamboa ${ }^{2}$, Miguel Briones-Salas ${ }^{1}$ \\ ${ }^{1}$ Laboratorio de Vertebrados Terrestres Centro Interdisciplinario de Investigación para el Desarrollo Integral Regional, Unidad \\ Oaxaca, Instituto Politécnico Nacional. Hornos 1003, CP. 71230, Santa Cruz Xoxocotlán, Oaxaca, México \\ ${ }^{2}$ Laboratorio de Sistemas de Información Geográfica. Instituto de Biología. Universidad Nacional Autónoma de México. \\ Circuito Exterior s/n, CP. 04510, Ciudad Universitaria, Coyoacán, Distrito Federal, México \\ ${ }^{*}$ Autor de correspondencia: mariolavnol@yahoo.com.mx
}

Artículo científico recibido: 24 de Julio de 2015, aceptado: 25 de febrero de 2016

RESUMEN. Oaxaca es el estado con mayor diversidad biológica de México, ocupa el primer lugar en riqueza de especies de anfibios, reptiles, aves y mamíferos. La pérdida y fragmentación del hábitat y la cacería excesiva son las principales amenazas en la conservación de estas especies. Para conocer el estado de conservación y normativo de los vertebrados terrestres del estado de Oaxaca, se consultó la Norma Oficial Mexicana 059, la lista roja de la Unión Internacional para la Conservación de la Naturaleza (UICN) y los apéndices de la Convención sobre el Comercio Internacional de Especies Amenazadas de Fauna y Flora Silvestres (CITES). De 1406 especies de vertebrados conocidos en Oaxaca, 580 (41.25 $\%$ ) se encuentran en alguna de las listas de conservación y normativas consultadas. En la Norma Oficial se encuentran 468 especies (80.68 \%), en la UICN 171 (29.43\%) y en los apéndices de CITES 160 (27.58 \%). De estas especies 100 son anfibios (17.24 \%), 152 reptiles $(26.20 \%), 254$ aves (43.79\%) y 74 mamíferos (12.75\%). La alta cantidad de especies con problemas de conservación, alertan para realizar mayores esfuerzos de conservación del patrimonio natural del estado.

Palabras clave: Anfibios, aves, categorías de riesgo, mamíferos, reptiles

ABSTRACT.The state of Oaxaca is one of the most biodiverse at Mexico; it occupies the first place of amphibians, reptiles, birds and mammals species richness. Nonetheless, habitat loss and fragmentation and overhunting are the main threats to species' conservation. In Mexico, NOM-059-SEMARNAT-2010 lists the flora and fauna species at risk. Globally, the red list of the International Union for Conservation of Nature (IUCN) catalogues the wild species with conservation problems and the Convention on International Trade in Endangered Species of Wild Fauna and Flora (CITES) rules the traffic of flora and fauna species. In order to know the conservation status of the state of Oaxaca, the risk categories of the terrestrial vertebrates with distribution in Oaxaca at the NOM-059-SEMARNAT-2010, the red list of the IUCN, and the CITES lists were consulted. We found a total of 580 terrestrial vertebrates in Oaxaca listed with a risk category; from these, we detected that 100 were amphibians (17.24 \%), 152 reptiles (26.20\%), 254 birds $(43.79 \%)$ and 74 mammals $(12.75 \%)$. The results showed that a high number of species has conservation threats and the rapidly demographic growth and the land use change in Oaxaca, enforce to conservation of the species in order to preserve the natural heritage of the state.

Key words: Amphibians, birds, mammals, reptiles, risk categories

\section{INTRODUCCIÓN}

La extinción de especies por causas antropogénicas es uno de los principales problemas que afectan a la diversidad biológica (Pimm y Brooks
1997). Con la pérdida de especies se eliminan relaciones interespecíficas, lo que provoca cambios en la dinámica del ecosistema; al mismo tiempo, el conocimiento local disminuye y se pierden soluciones de problemas actuales y futuros (Díaz et al. 
2006). Las principales causas de la extinción de especies son la pérdida y fragmentación del hábitat, la cacería excesiva, la explotación de las poblaciones y la presencia de especies exóticas (Álvarez-Romero et al. 2008). Particularmente en México, en los últimos dos siglos, 43 vertebrados se han extinguido (Ceballos et al. 2005), se estima que en los próximos 100 años entre el 10 y $50 \%$ de la biodiversidad del mundo se perderá (Mace et al. 2006).

Por la preocupación por la extinción de la flora y fauna, la Unión Internacional para la Conservación de la Naturaleza publicó un registro de la vida silvestre amenazada (Collar 1996). En México Villa-Ramírez (1978) proporcionó la primera lista de las especies de vertebrados raros y en peligro de extinción. Posteriormente Ceballos y Navarro (1991) evaluaron el riesgo de extinción de los mamíferos terrestres mexicanos. Estos estudios fueron la base de la Norma Oficial Mexicana NOM-059-ECOL1994, que listó 2423 especies, 934 hongos y plantas y 1389 animales (SEDESOL 1994). En la renovación del 2002 de la Norma Oficial Mexicana 059, se mencionan 2477 especies, las especies en la categoria rara se reubicaron como sujetas a protección especial de manera precautoria, hasta que se cuente con mayor información para determinar su situación. Otro avance fue el establecimiento del Método de Evaluación de Riesgo de Extinción de Especies Silvestres en México (MER), basado en criterios de distribución, estado del hábitat, vulnerabilidad biológica e impacto de la actividad humana (SEMARNAT 2002). En el 2010 se publicó la tercera edición de la NOM-059-SEMARNAT2010 en la que se determina el riesgo de extinción (SEMARNAT 2010). La lista roja de la Unión Internacional para la Conservación de la Naturaleza (IUCN) y los apéndices de la Convención sobre el Comercio Internacional de Especies Amenazadas de Fauna y Flora Silvestres (CITES) son las listas recurridas para determinar el riesgo de extinción de la fauna y flora (Mace et al. 2006, CITES 2015). El estado de Oaxaca es uno de los que tiene mayor diversidad biológica de México (García-Mendoza et al. 2004), y se encuentra en una de las ecorregiones de prioridad global para la conservación (Olson y
Dinerstein 2002); ocupa los primeros lugares en riqueza de especies de anfibios, reptiles, aves y mamíferos (Navarro-Sigüenza et al. 2014, BrionesSalas et al. 2015a). Por ello el objetivo del trabajo fue analizar el estado de conservación de las especies de anfibios, reptiles, aves y mamíferos del estado de Oaxaca.

\section{MATERIALES Y MÉTODOS}

Se actualizaron las listas de especies de anfibios, reptiles, aves y mamíferos del estado de Oaxaca, tomando como referencia los trabajos de Casas-Andreu et al. (2004), Navarro-Sigüenza et al. (2004) y Briones-Salas y Sánchez-Cordero (2004). Los vertebrados terrestres fueron todos aquellos tetrápodos que viven en el ecosistema terrestre (Long y Gordon 2004).

Se realizó una búsqueda en internet de publicaciones que tratan sobre la descripción de nuevas especies, nuevos registros para el estado, cambios y arreglos nomenclaturales y taxonómicos, tomando como buscador la página del Google Scholar indicando las palabras clave en español e inglés: nuevo registro, nueva especie, revisión taxonómica, anfibio, reptil, ave, mamífero y Oaxaca, deliminanto las publicaciones para el período comprendido entre el 2004 y 2015.

También se consultó las listas de especies en riesgo en la Norma Oficial Mexicana NOM-059SEMARNAT-2010, la lista roja de la IUCN y los apéndices CITES. Se contabilizó el número de especies en cada categoría y lista, para calcular el número de especies en cada categoria y determinar el porcentaje con respecto a la riqueza específica por grupo y total.

\section{RESULTADOS}

La riqueza de vertebrados terrestres nativos de Oaxaca es de 1406 especies, de las que 154 $(10.95 \%)$ son anfibios, $292(20.76 \%)$ reptiles, 744 (52.91\%) aves y $216(15.36 \%)$ mamíferos. De las cuales 580 son especies en riesgo en alguna de las listas, misma que representa el $41.25 \%$ de las 


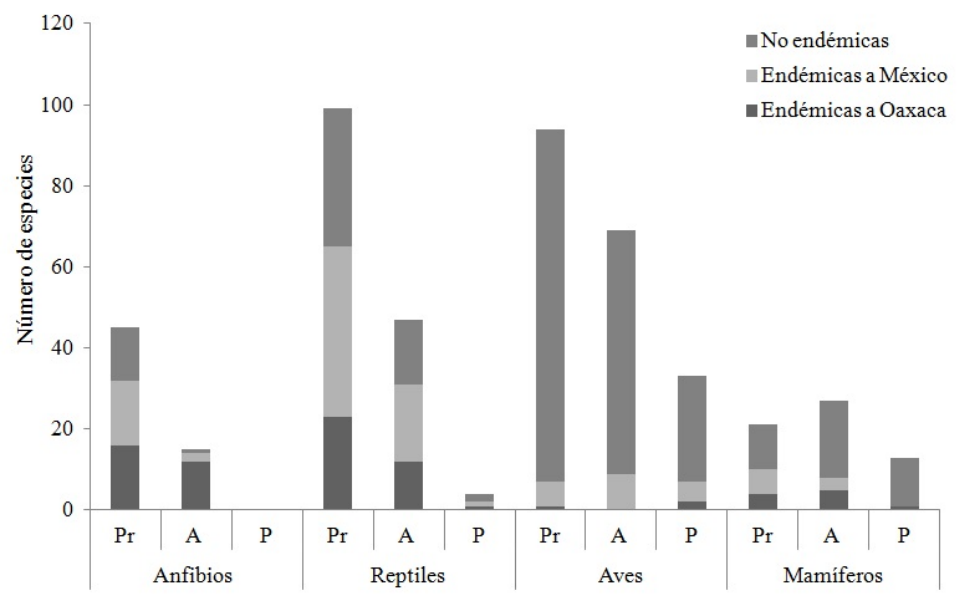

Figura 1. Número de especies de vertebrados terrestres de Oaxaca en categorías de riesgo de la Norma Oficial Mexicana NOM-059-SEMARNAT-2010. Categorías: Pr, sujetas a protección especial; A, amenazadas; $\mathrm{P}$, en peligro de extinción.

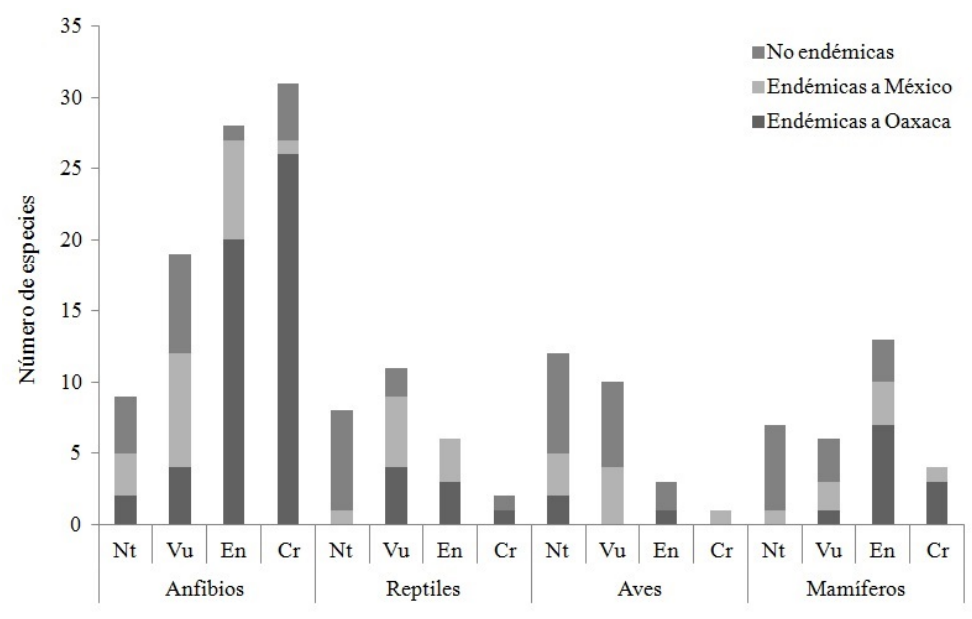

Figura 2. Número de especies de vertebrados terrestres de Oaxaca en categorías de riesgo de la lista roja de la Unión Internacional para la Conservación de la Naturaleza. Categorías: $\mathrm{Nt}$, casi amenazada; $\mathrm{Vu}$, vulnerable; En, en peligro; $\mathrm{Cr}$, peligro crítico.

especies reportadas en el estado. En la NOM-059SEMARNAT-2010 se encuentran 468 especies, en la lista roja de la IUCN 171 y en los apéndices CITES 160. Para los anfibios el $64.93 \%$ (100) de las especies se encuentran en alguna categoría de riesgo, de las cuales 75 están sujetas a protección especial y 25 amenazadas (Figura 1), 87 en la lista roja de la IUCN y una especie en los apéndices de la CITES, de estas 23 especies son endémicas de México. En la lista roja de la IUCN el $67.81 \%$ de los anfibios se encuentra en peligro y en peligro crítico (Figura
2). Del orden Anura y Caudata, 21 y 10 especies están en peligro crítico, respectivamente. De Anura 16 especies y todas las especies de Caudata son endémicas de Oaxaca. En los anfibios listados por la CITES, sólo una especie se encuentra en Oaxaca (Figura 3).

Ciento cincuenta y dos especies de reptiles se encuentran en alguna categoría de riesgo, lo que representa el $52.05 \%$ de la riqueza de reptiles del estado. De las especies en riesgo, 150 estan en la NOM-059-SEMARNAT-2010, 27 en la IUCN y 17 


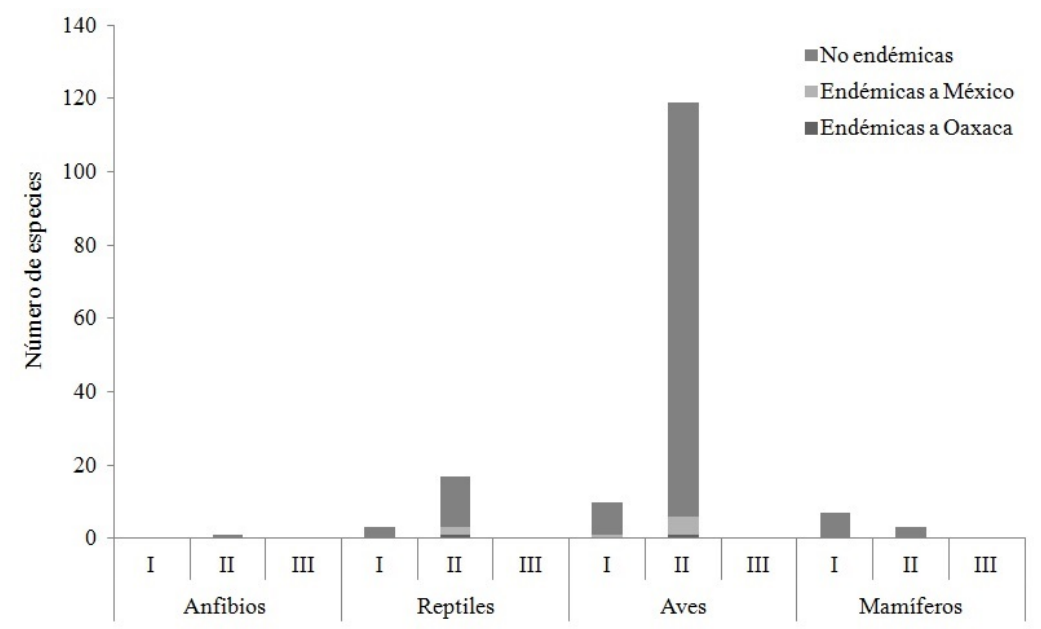

Figura 3. Número de especies de vertebrados terrestres de Oaxaca en los apéndices de la Convención sobre el Comercio Internacional de Especies Amenazadas de Fauna y Flora Silvestres.

en los apéndices CITES. De las cuales 100 especies son endémicas de México y 37 del estado de Oaxaca.

De las especies de reptiles listadas en la NOM-059-SEMARNAT-2010, 99 están sujetas a protección especial, 47 se encuentran amenazadas y cuatro en peligro de extinción (Figura 1). Mientras que la IUCN cataloga a dos especies en peligro crítico, seis en peligro y ocho como casi amenazadas (Figura 2). En tanto que en los apéndices CITES, una especie se encuentra en el apéndice I y 16 en el apéndice II (Figura 3).

Para aves se tienen 254 especies en alguna de las listas, lo que representa el $34.14 \%$ de las especies del estado de Oaxaca. De estas, 25 especies son endémicas de México y tres de Oaxaca. De las cuales 196 se encuentran protegidas en la NOM059-SEMARNAT-2010, 26 en riesgo en la IUCN y 129 en los apéndices CITES. En la NOM-059SEMARNAT-2010, 94 especies de aves estan sujetas a protección especial, 69 amenazadas y 33 en peligro de extinción (Figura 1). Mientras que la lista roja de la IUCN incluye 12 especies de aves como casi amenazadas, 10 como vulnerables, 3 en peligro y Puffinus auricularis en peligro crítico (Figura 2). En los apéndices CITES, 10 especies se encuentran en el apéndice I y 119 en el apéndice II (Figura 3). La CITES incluye en el apéndice II a las especies de los ordenes Accipitriformes, Falconiformes, Psitaciformes, Strigiformes, y a la familia Trochilidae.

En total 74 especies de mamíferos se encuentran catalogadas en riesgo de conservación, lo que representa el $35.25 \%$ de los mamíferos registrados en el estado. La NOM-059-SEMARNAT-2010 incluye 61 , mientras que la IUCN reconoce 31 mamíferos en riesgo y en los apéndices CITES 10 especies. De las cuales 25 son endémicas, 12 de México y 13 de Oaxaca. En la NOM-059SEMARNAT-2010, 21 especies se encuentran sujetas a protección especial, 27 amenazadas, 13 en peligro de extinción y una extinta en vida silvestre (Figura 1). En la lista roja de la IUCN cuatro especies están en peligro crítico, 13 en peligro, siete casi amenazadas, seis vulnerables, y una está extinta en vida silvestre (Figura 2). La NOM-059SEMARNAT-2010 y la IUCN consideran a Canis lupus baileyi como extinta en vida silvestre. Mientras que la CITES incluye 10 especies, tres en el apéndice II y siete especies en el apéndice I (Figura 3).

\section{DISCUSIÓN}

Se obtuvo la relación de las especies de vertebrados terrestres en riesgo o sujetas a regulación 


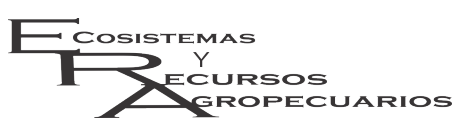

del estado de Oaxaca. Para medir la vulnerabilidad ambiental de los anfibios y reptiles Wilson et al. (2013a, 2013b) proponen el uso de la extensión de su distribución geográfica, la distribución ecológica y el modo de reproducción; mientras que Quintero et al. (2014) proponen una estimación de las tendencias poblacionales de los anfibios mexicanos, considerados con datos insuficientes por la IUCN. Al respecto Collen et al. (2011) proponen la inclusión de la distinción filogenética para priorizar la conservación global de mamíferos. En aves, Panjabi et al. (2012) indican sólo el uso de criterios biológicos. Por lo que es evidente que los distintos grupos de especies requieren ser evaluados con criterios acordes a sus atributos ecológicos y amenazas. De las 1406 especies de vertebrados, 580 especies se encuentran amenazadas y sujetas a regulación en el estado de Oaxaca, lo que representa el $41.25 \%$ de las especies. La mayoría de estas especies se encuentran en la NOM-059-SEMARNAT-2010, con menor proporción en la lista roja de la IUCN y en los apéndices CITES. Otras comparaciones entre las listas de especies amenazadas, han reportado discrepancias en el número de especies y categorías de riesgo, que se pueden atribuir a los objetivos, aspectos taxónomicos o efectos de escala geográfica (Brito et al. 2015).

En la publicación del 2015 de la IUCN, se incluyen 1994 especies de anfibios como amenazadas, 944 reptiles, 1375 aves y 1197 mamíferos (IUCN 2015). Mientras que en la NOM-059-SEMARNAT2010 se encuentran 94 especies de anfibios, 438 de reptiles, 297 de aves y 157 de mamíferos en alguna categoría de amenaza (SEMARNAT 2010). Por lo que en Oaxaca se encuentra el 4.36, 2.86, 1.89 y $2.25 \%$ de las especies de anfibios, reptiles, aves y mamíferos terrestres amenazados a nivel mundial y el $30.92,34.96,65.99$ y el $38.85 \%$ de las especies de estos grupos en alguna categoría de riesgo en la NOM-059-SEMARNAT-2010.

En la evaluación del estatus de conservación de los anfibios mexicanos, la IUCN reporta que el $58.00 \%$ de los anfibios del país están amenazados (Frías-Alvarez et al. 2010). Mientras que la NOM-059-SEMARNAT-2010 incluye 194 especies
Lavariega et al.

Vertebrados amenazados en Oaxaca, México Esosist. Recur. Agropec. 4(10):135-146,2017

de anfibios (SEMARNAT 2010), lo que representa el $51.60 \%$ de las especies conocidas en el país (ParraOlea et al. 2014). De las especies de anfibios conocidas en el estado de Oaxaca en el 2004, el 44.40 $\%$ (59) se encontraban en la NOM-059-ECOL-2001 (Casas-Andreu et al. 2004), incrementando a 60 en el presente estudio. El aumento de especies en las categorías de riesgo se debe a la revalorización de las especies ya al aumento en el conocimiento de la riqueza específica de este grupo.

Los anfibios son el grupo de vertebrados más amenazado a nivel mundial, con cerca de la mitad de las especies en vías de extinción, principalmente por la pérdida del hábitat, la sobreexplotación, enfermedades y cambios en el clima (Stuart et al. 2004). Pruebas del decline de las poblaciones de anfibios por enfermedades en el sur de México fueron documentas por Lips et al. (2004), que en hábitats en buen estado de conservación del estado de Oaxaca, encontraron 27 especies de anfibios con poblaciones disminuidas. También indicaron que es probable que 11 especies esten extintas, además de evidencias de anfibios infectados por Batrachochytridium dendrobatidis. Otra amenaza a la diversidad de anfibios es el cambio climático, se estima que la riqueza de especies disminuirá de 333 en 2000 , a 300 en 2020, 258 en 2050 y 254 en 2050 (Ochoa-Ochoa et al. 2012). A pesar de ello, la representación de epecies de anfibios amenazados es baja en el sistema de áreas naturales protegidas (ANP) de Oaxaca, con una especie en el Monumento Natural Yagul, cinco en el Parque Nacional Huatulco, cinco en la Reserva de la Biosfera Tehuacán-Cuicatlán, siete en el Área de Protección de Flora y Fauna Boquerón de Tonalá y 10 en el Parque Nacional Benito Juárez (CONANP 2003, SEMARNAT 2013a, 2013b, 2013c, 2013e).

En México se reportan 849 especies de reptiles (Wilson et al. 2013b), de las cuales la NOM059-SEMARNAT-2010 lista 438 especies, 273 sujetas a protección especial, 138 amenazadas y 27 en peligro de extinción (SEMARNAT 2010) y en la lista roja de la IUCN se reportan 118 especies (Wilson et al. 2013b). Hasta el 2004, se conocían 239 especies de reptiles terrestres en Oaxaca, de las que el $51.88 \%$ estaban en riesgo (Casas-Andreu et 
al. 2004). Actualmente, se reportan 292 especies, de las cuales el $51.37 \%$ están en la NOM-059SEMARNAT-2010. Al igual que en otros grupos de vertebrados terrestres, la destrucción del hábitat, el tráfico de especies, las especies invasoras, la contaminación, las enfermedades y el cambio climático son las principales causas que perjudican a las poblaciones de reptiles (Böhm et al. 2013). En el estado de Oaxaca, se añade la idea de que los animales son nocivos, repugnantes y/o peligrosos, lo que dificulta su protección (Aranda-Coello y Ochoa-Ochoa 2015).

La representación de reptiles en las ANP del estado de Oaxaca es más favorable, con siete especies en el Monumento Natural Yagul, 13 en el Parque Nacional Lagunas de Chacahua, 18 en el Área de Protección de Flora y Fauna Boquerón de Tonalá, 20 en el Parque Nacional Benito Juárez, 40 en el Parque Nacional Huatulco y 45 en la Reserva de la Biosfera Tehuacán-Cuicatlán (CONANP 2003, SEMARNAT 2013a, 2013b, 2013c, 2013e). Otros sitios con protección federal en el Oaxaca son las zonas arqueológicas del Cerro Guiengola, Istmo de Tehuantepec, en donde más de la mitad de las especies registradas se encuentran en alguna categoria de riesgo (Martín-Regalado et al. 2011). Para los anfibios y reptiles, el número de especies conocidas en Oaxaca entre 2004 y 2015 tuvo un aumento de 133 a 154 y de 245 a 297 especies, respectivamente. La mayoría de las nuevas especies no han sido evaluadas, por lo que es posible que el número de especies en riesgo incremente, particularmente por ser especies con distribución geográfica restringida con hábitats bajo presión antrópica (Mata-Silva et al. 2015).

De las 1123 especies de aves reportadas en México (Navarro-Sigüenza et al. 2014), la NOM-059-SEMARNAT-2010 reporta 297 especies en alguna categoria de riesgo (SEMARNAT 2010). Mientras que Navarro-Sigüenza et al. (2014) indican que 114 especies de aves presentes en el estado de Oaxaca están en la lista roja de la IUCN y 140 en los apéndices CITES. La NOM-059-ECOL-2001 reporta 195 especies de aves en riesgo (NavarroSigüenza et al. 2002), mientras que norma NOM-
059-SEMARNAT-2010 reporta 196, disminuyendo las especies sujetas a protección especial de 114 a 94, y aumentando las especies amenazadas de 58 a 69 y en peligro de extinción de 23 a 33 (SEMARNAT 2010). Con respecto a la IUCN, el número de especies casi amenazadas, en peligro crítico y en peligro se mantiene igual, mientras que las especies vulnerables pasan de 8 a 10 (IUCN 2015). Entre las principales amenazas para la conservación de las aves en México están la pérdida y degradación del hábitat, tráfico y comercio, cacería excesiva, contaminación, especies introducidas y alteración de regímenes naturales (Îñigo-Elías y Enkerlin-Hoeflich 2002). Hasta el 2002, 24 especies y subespecies se consideraban extintas en México, de las cuales Ibycter americanus, Ectopistes migratorius y Sporophila schistacea se encontraban en Oaxaca (Ríos-Muñoz 2002). Actualmente, 33 se consideran en peligro de extinción por la NOM-059-SEMARNAT-2010 y cuatro en peligro o en peligro crítico por la IUCN (SEMARNAT 2010, IUCN 2015). A nivel mundial, la situación de las aves amenazadas continua en deterioro, con un declive mayor en las aves de presa y cinegéticas (Butchart et al. 2004). En Oaxaca, las aves de los órdenes Psittaciformes son capturadas para el comercio como mascotas, los Accipitriformes y Falconiformes para cetrería y los Galliformes para alimento. A pesar de la situación de estos órdenes, existe poca información de sus poblaciones, sólo de Ara militaris se conoce la abundancia estacional y cronología reproductiva (Rivera-Ortíz et al. 2008). Por la frecuencia de captura para el comercio ilegal para el comercio, ha ocasionado que Amazona auropalliata, $A$. farinosa y Brotogeris jugularis se consideren extintas en el estado (Cantú et al. 2007). Estimandose una captura anual de 15000 individuos, lo que coloca a la entidad en el primer lugar. Un reto para la conservación de aves es disminuir su demanda y captura (Wright et al. 2001). Sobre esto Navarro-Sigüenza y Sánchez-González (2002) identificaron 15 áreas importantes para la conservación de aves en México, entre las cuales se encuentran la Reserva de la Biosfera TehuacánCuicatlán, el Parque Nacional Lagunas de Chacahua y el Parque Nacional Benito Juárez. 
A nivel nacional, la NOM-059-SEMARNAT2010 reporta 157 especies de mamíferos terrestres en peligro, de las 496 (Ramírez-Pulido et al. 2014). Al contrastar la versión de la NOM-059 del 2001 y 2010, 50 de las 190 especies de mamíferos del estado de Oaxaca estan en alguna categoría de riesgo, de las cuales 17 estan sujetas a protección especial, 21 amenazadas y 12 en peligro de extinción (BrionesSalas y Sánchez-Cordero 2004). Mientras que en la NOM-059-SEMARNAT-2010, el número de especies sujetas a protección especial aumentan a 21 , las amenazadas a 27 y las que están en peligro de extinción a 13. Al respecto Briones-Salas y SánchezCordero (2004) indican que 45 especies estaban bajo alguna categoria de riesgo por la IUCN, de las cuales 26 estan casi amenazadas, 14 vulnerables, cuatro en peligro, y una extinta en vida silvestre. En la presente revisión, el número de mamíferos terrestres en las categorías de riesgo de la IUCN fue de siete casi amenazadas, seis vulnerables, 13 en peligro, y cuatro en peligro crítico. En los apéndices CITES se encuentran 10 en el apéndice I, cinco en el apéndice II, y 10 en el apéndice III (Briones-Salas y SánchezCordero 20004). Mientras que el útlimo reporte CITES El número de especies en los apéndices disminuyó a siete en el apéndice I y tres en el apéndice II (CITES 2015).

En México, los estados más ricos en biodiversidad estan en el sureste (Sánchez-Cordero et al. 2014). En los que hay un alto número de especies en riesgo (Ceballos et al. 2005). Al Respecto, Schipper et al. (2008) indican que las amanazas globales para los mamíferos son la pérdida y degradación del hábitat, sobrexplotación, contaminación, mortalidad accidental, enfermedades y especies exóticas invasoras. Con mayor vulnerabilidad las especies con distribución geográfica reducida, con bajas tasas reproductivas y talla corporal grande (Davidson et al. 2008). Además, éstas últimas suelen ser de las especies más explotadas (Cardillo et al. 2005).

En el estado de Oaxaca, algunas especies reportadas en la NOM-059-SEMARNAT-2010 han sido reportadas en el Sistema Nacional de Áreas Naturales Protegidas (SINAP), dos en el Monumento Natural Yagul, seis en el Área de Proteccion de Flora y Fauna Boquerón de Tonalá, nueve en el Parque Nacional Benito Juárez, doce en el Parque Nacional Lagunas de Chacahua, 18 en la Reserva de la Biosfera Tehuacán-Cuicatlán y 24 en el Parque Nacional Huatulco (CONANP 2003, SEMARNAT 2013a, 2013b, 2013c). Pero hace falta mayores esfuerzos para inventariar estas áreas protegidas, así como otras de iniciativa comunitaria para conocer el estado de las poblaciones de mamíferos (BrionesSalas et al. 2015b).

En general se encontraron cambios en el número de especies catalogadas en riesgo con respecto a revisiones anteriores (Briones-Salas y Sánchez-Cordero et al. 2004, Navarro-Sigüenza et al. 2004). Aunque los cambios entre evaluaciones de distintos años pueden arrojar interpretaciones erróneas de la tendencia de la situación de riesgo de las especies, debido a que el conocimiento de ellas aumenta con el tiempo (Butchart et al. 2004, Vilela et al. 2014).

A nivel nacional, la política pública dirigida a la conservación y manejo de especies en peligro de extinción es el Programa de Conservación de Especies en Riesgo (PROCER), que incluye a 40 especies de animales, de las cuales las aves Aquila chrysaetus, Ara militaris, Ara macao, Amazona auropalliata, A. oratrix, Harpia harpyja, Spizaetus ornatus, S. tyrannus, Spizastur melanoleucus, y los mamíferos Panthera onca, Tapirella bairdii, Ateles geoffroyi, Alouatta palliata, Tayassu pecari, Leptonycteris nivalis y $L$. yerbabuenae se encuentran en Oaxaca. Además de Canis lupus baileyi, especie extinta en vida silvestre (SEMARNAT 2012). Cada especie incluida en el PROCER cuenta con un Programa de Acción para la Conservación de Especies. El gobierno mexicano establece como estrategias principales el fomento a la conservación y restauración de los ecosistemas, su biodiversidad y la conservación de las especies en riesgo (SEMARNAT 2013d). Si bien el número de especies de vertebrados indicadas en el PROCER representa una fracción (3.71\%) del total de especies en la NOM059-SEMARNAT-2010, los recursos designados a la implementación de acciones para su conservación pueden justificarse si se usan de forma racional en 
términos del costo y éxito de su implementación (Joseph et al. 2009). En Oaxaca es relevante la conservación de los bosques y selvas por parte de comunidades indígenas, lo que ha contribuido en la conservación de la biodiversidad (Martin et al. 2010). Sin embargo, estas áreas pueden estar separadas por lo que se deben desarrolar políticas y acciones de conservación para asegurar la conectividad entre fragmentos de hábitat aislados (Reed 2004). Muchas especies amenazadas con poblaciones en el estado de Oaxaca, ya están extintas en los estados vecinos, lo que indica la importancia de su conservación. Lo que representa un reto para su protección, debido a los actuales ritmos de cambio de uso de suelo de la entidad. Por ello es importante conocer su distribución geográfica, la condición del hábitat, la situación de sus poblaciones y las amenazas locales (Rodriguez 2008).

\section{CONCLUSIONES}

En el estado de Oaxaca habitan 1406 es- pecies de vertebrados terrestres, de las cuales 154 son anfibios, 292 reptiles, 744 aves y 216 mamíferos, lo que coloca a la entidad en el primer lugar en riqueza de especies. Del total, 580 se encuentran en alguna categoría de riesgo o regulación; la mayor cantidad en la NOM-059-SEMARNAT-2010 (80.68 $\%)$, seguida por la lista roja de la IUCN (29.43\%) y los apéndices CITES (27.58 \%). De las especies en riesgo, 96 son anfibios, 143 reptiles, 254 aves y 74 mamíferos.

\section{AGRADECIMIENTOS}

A los revisores anónimos y el editor en jefe que realizaron observaciones y comentarios que ayudaron a mejorar el manuscrito. El MC. Lavariega agradece el apoyo de la beca CONACYT y PIFIIPN, para estudios de maestría. M. Briones-Salas agradece a EDI y COFAA del IPN, y al SNI el apoyo económico y reconocimiento.

\section{LITERATURA CITADA}

Álvarez-Romero JG, Medellín RA, Oliveras De Ita A, Gómez De Silva H, Sánchez O (2008) Animales exóticos en México: una amenaza para la biodiversidad. Comisión Nacional para el Conocimiento y Uso de la Biodiversidad, Instituto de Ecología, UNAM, SEMARNAT. México, DF. 518p.

Aranda-Coello M, Ochoa-Ochoa LM (2015) Tres meses de rescate de anfibios y reptiles en una localidad del sur de Oaxaca, México. Revista Mexicana de Herpetología 1: 22-24.

Böhm M, Collen B, Baillie JEM, Bowles P, Chanson J, Cox N, et al. (2013) The conservation status of the world's reptiles. Biological Conservation 157: 372-385.

Briones-Salas M, Cortés-Marcial M, Lavariega MC (2015a) Diversidad y distribución geográfica de los mamíferos terrestres del estado de Oaxaca, México. Revista Mexicana de Biodiversidad 86: 685-710.

Briones-Salas M, Lavariega MC, Cortés-Marcial M, Monroy-Gamboa AG, Mases-García CA (2015b) Iniciativas de conservación para los mamíferos de Oaxaca, México. En: Briones-Salas M, Hortelano-Moncada Y, Magaña-Cota G, Sánchez-Rojas G, Sosa-Escalante JE (eds). Riqueza y conservación de los Mamíferos en México a nivel estatal. Instituto de Biología UNAM, AMMAC, UG. México, DF. pp: 289-325.

Briones-Salas M, Sánchez-Cordero V (2004) Mamíferos. In: García Mendoza AJ, Ordóñez MJ, Briones-Salas M (eds) Biodiversidad de Oaxaca. Instituto de Biología, UNAM, Fondo Oaxaqueño para la Conservación de la Naturaleza-World Wildlife Fund. México, DF. pp: 423-447.

Brito D, Ambal RG, Brooks T, De Silva N, Foster M, Hao W, et al. (2015) How similar are national red lists and the IUCN Red List? Biological Conservation 143: 1154-1158. 
Butchart SH, Stattersfield AJ, Bennun LA, Shutes SM, Akcakaya HR, Baillie JEM, et al. (2004) Measuring global trends in the status of biodiversity: Red List Indices for Birds. PLoS Biology 2: 1-11.

Cantú JC, Sánchez ME, Grosselet M, Silva J (2007) Tráfico ilegal de pericos en México: una evaluación detallada. Defenders of Wildlife, Teyeliz, México, DF. 80p.

Cardillo M, Mace GM, Jones KE, Bielby J, Bininda-Emonds ORP, Sechrest W, et al. (2005) Multiple causes of high extinction risk in large mammal species. Science 309: 1239-1241.

Casas-Andreu G, Méndez-De La Cruz FR, Aguilar-Miguel X (2004) Anfibios y reptiles. In: García Mendoza AJ, Ordóñez MJ, Briones-Salas M (eds) Biodiversidad de Oaxaca. Instituto de Biología, UNAM, Fondo Oaxaqueño para la Conservación de la Naturaleza-World Wildlife Fund. México, DF. pp: 375-390.

Ceballos G, Navarro D (1991) Diversity and conservation of mexican mammals. In: Mares MA, Schmidly DJ (eds.) Latin American Mammalogy. History, Biodiversity and Conservation. University of Oklahoma Press, Oklahoma. 473p.

Ceballos G, Oliva G (2005) Los mamíferos silvestres de México. Fondo de Cultura Económica, Comisión Nacional para el Conocimiento y Uso de la Biodiversidad. México, DF. 986p.

CITES (2015) Appendices I, II, and III. Convention on International Trade in Endangered Species of Wild Fauna and Flora, UNEP. Ginebra, Suiza. 46p.

Collar NJ (1996) The reasons for Red Data Books. Oryx 30: 121-130.

Collen B, Turvey ST, Waterman C, Meredith HMR, Kuhn TS, Baillie JEM, et al. (2011) Investing in evolutionary history: implementing a phylogenetic approach for mammal conservation. Philosophical Transactions of The Royal Society 366: 2611-2622.

CONANP (2003) Programa de Manejo Parque Nacional Huatulco. Comisión Nacional de Áreas Naturales Protegidas. México, DF. 209p.

Davidson AD, Hamilton MJ, Boyer AG, Brown JH, Ceballos G (2008) Multiple ecological pathways to extinction in mammals. Proceedings of the National Academy of Sciences 106: 10702-10705.

Díaz S, Fargione J, Chaplin III FS, Tilman D (2006) Biodiversity loss threatens human well-being. PLOS Biology 4: 1300-1305.

Frías-Alvarez P, Zúñiga-Vega JJ, Flores-Villela O (2010) A general assessment of the conservation status and decline trends of Mexican amphibians. Biodiversity Conservation 19: 3699-3742.

García-Mendoza AJ, Ordóñez MJ, Briones-Salas M (2004) Biodiversidad de Oaxaca. Instituto de Biología, UNAM-FOCN. México, DF. 605p.

Íñigo-Elías EE, Enkerlin-Hoeflich EC (2002) Amenazas, estrategias e instrumentos para la conservación de las aves. En: Gómez De Silva H, Oliveras De Ita A (eds) Conservación de aves, experiencias en México. CIPAMEX, CONABIO, NFWF. México DF. pp: 86-132.

IUCN (2015) IUCN Red List of Threatened Species. Version 2015.4. http://www.iucnredlist.org/. Fecha de consulta 20 de noviembre de 2015.

Joseph LN, Maloney RF, Possingham HP (2009) Optimal allocation of resources among threatened species: a project priorization protocol. Conservation Biology 23: 328-338.

Lips KR, Mendelson III JR, Muñoz-Alonso A, Canseco-Márquez L, Mulcahy DG (2004) Amphibian population declines in montane southern Mexico: resurveys of historical localities. Biological Conservation 119: 555-564. 
Long JA, Gordon MS (2004) The greatest step in vertebrate history: a paleobiological review of the fishtetrapod transition. Physiological and Biochemical Zoology: Ecological and Evolutionary Approaches 77:700-719.

Mace GM, Possingham HP, Leader-Williams N (2006) Prioritizing choices in conservation. In: Macdonald DW, Srvice K (eds). Key Topics in Conservation Biology. Blackwell Publishing. Massachusetts. pp: 17-34.

Mata-Silva V, Johnson JD, Wilson LD, García-Padilla E (2015) The herpetofauna of Oaxaca, Mexico: composition, physiographic distribution, and conservation status. Mesoamerican Herpetology 2: 1-62.

Martin GJ, Camacho CI, Del Campo CA, Anta S, Chapela F, González MA (2010) Indigenous and community conserved areas in Oaxaca, Mexico. Management of Environmental Quality: An International Journal 22: $250-266$.

Martín-Regalado CN, Gómez-Ugalde RM, Cisneros-Palacios M (2011) Herpetofauna del Cerro Guiengola, Istmo de Tehuantepec, Oaxaca. Acta Zoológica Mexicana 27: 359-376.

Navarro-Sigüenza AG, García-Trejo EA, Peterson AT, Rodríguez-Contreras V (2004). Aves. In: García AJ, Ordóñez MJ, Briones-Salas M (eds) Biodiversidad de Oaxaca. Instituto de Biología, UNAM, FOCN WWF. México, DF. pp: 391-421.

Navarro-Sigüenza AG, Rebón-Gallardo MF, Gordillo-Martínez A, Peterson AT, Berlanga-García H, SánchezGonzález LA (2014) Biodiversidad de aves en México. Revista Mexicana de Biodiversidad, Suplemento 85: S476-S495.

Navarro-Sigüenza AG, Sánchez-González LA (2002) La diversidad de aves. In: Gómez De Silva H, Oliveras De Ita A (eds) Conservación de aves, experiencias en México. CIPAMEX. México DF. pp: 24-56.

Ochoa-Ochoa LM, Rodríguez P, Mora F, Flores-Villela O, Whittaker RJ (2012) Climate change and amphibian diversity patterns in Mexico. Biological Conservation 150: 94-102.

Olson DM, Dinerstein E (2002) The global 200: Priority ecoregions for global conservation. Annals of Missouri Botanical Garden 89: 199-224.

Panjabi AO, Blancher PJ, Dettmers R, Rosenberg KV (2012) Partners in Flight Technical Series No. 3. Rocky Mountain Bird Observatory website: http://www.rmbo.org/pubs/downloads/Handbook2012.pdf. Fecha de consulta 5 de julio de 2015.

Parra-Olea G, Flores-Villela O, Mendoza-Almerall C (2014) Biodiversidad de anfibios en México. Revista Mexicana de Biodiversidad, Suplemento 85: S460-S466.

Pimm SL, Brooks TM (1997) The sixth extintion: How large, how soon, and where? In: Rave PH (ed). Nature and human society, the quest for a sustainable world. National Academy Press. Washington, DC. pp: 46-62.

Quintero E, Thessen AE, Arias-Caballero P, Ayala-Orozco B (2014) A statistical assessment of population trends for data deficient Mexican amphibians. PeerJ2:e703.

Ramírez-Pulido J, González-Ruiz N, Gardner AL, Arroyo-Cabrales J (2014) List of recent land mammals of Mexico, 2014. Special Publications Museum of Texas Tech University 63: 1-76.

Reed DH (2004) Extinction risk in fragmented habitats. Animal Conservation 7: 181-191.

Ríos-Muñoz CA (2002) La diversidad perdida: las aves desaparecidas de México. En: Gómez De Silva H, Oliveras De Ita A (eds). Conservación de aves, experiencias en México. Sociedad para el Estudio y Conservación de las Aves de México. México, DF. pp: 69-85. 
Rivera-Ortíz F, Contreras-González AM, Soberanes-González SA, Valiente-Banuet A, Arizmendi MC (2008) Seasonal abundance and breeding chronology of the military macaw (Ara militaris) in a semi-arid region of central Mexico. Ornitología Neotropical 19: 255-263.

Rodriguez JP (2008) National Red List: the largest global market for IUCN Red List Categories and Criteria. Endangered Species Research 6: 193-198.

Sánchez-Cordero V, Botello F, Flores-Martínez JJ, Gómez-Rodríguez RA, Guevara L, Gutiérrez-Granados G, et al. (2014) Biodiversidad de Chordata (Mammalia) en México. Revista Mexicana de Biodiversidad, Suplemento 85: S496-S504.

Schipper J, Chanson JS, Chiozza F, Cox NA, Hoffmann M, KAtariya V, et al. (2008) The status of the world's land and marine mammals: diversity, threat, and knowledge. Science 322: 225-230.

SEDESOL (1994) Norma Oficial Mexicana NOM-059-ECOL-1994, que determina las especies y subespecies de flora y fauna silvestres terrestres y acuáticas en peligro de extinción, amenazadas, raras y sujetas a protección especial y que establece especificaciones para su protección. Diario Oficial de la Federación 438: $2-60$.

SEMARNAT (2002) Norma Oficial Mexicana NOM-059-ECOL-2001, Protección ambiental-especies nativas de México de flora y fauna silvestres-categorías de riesgo y especificaciones para su inclusión, exclusión o cambio-lista de especies en riesgo. Diario Oficial de la Federación 2288: 1-85.

SEMARNAT (2010) Norma Oficial Mexicana NOM-059-SEMARNAT-2010, Protección ambiental-especies nativas de México de flora y fauna silvestres-categorías de riesgo y especificaciones para su inclusión, exclusión o cambio-lista de especies en riesgo. Diario Oficial de la Federación 2454: 1-77.

SEMARNAT (2012) Programa de Conservación de Especies en Riesgo PROCER 2007-2012. http://procer.co nanp.gob.mx/pdf/procer_2012.pdf

SEMARNAT (2013a) Programa de Manejo Área de Protección de Flora y Fauna Boquerón de Tonalá. Secretaria de Medio Ambiente y Recursos Naturales. México, DF. 156p.

SEMARNAT (2013b) Programa de Manejo Monumento Natural Yagul. Secretaria de Medio Ambiente y Recursos Naturales. México, DF. 142p.

SEMARNAT (2013c) Programa de Manejo Reserva de la Biosfera Tehuacán-Cuicatlán. Secretaria de Medio Ambiente y Recursos Naturales. México, DF. 336p.

SEMARNAT (2013d) Programa sectorial del medio ambiente y recursos naturales 2013-2018 Resumen ejecutivo. Secretaria de Medio Ambiente y Recursos Naturales. México, DF. Fecha de consulta 20 de noviembre de 2015. http://www.semarnat.gob.mx/si

tes/default/files/documentos/transparencia/promarnat_resumen_ejecutivo_final.pdf

SEMARNAT (2013e) Resumen del Programa de Manejo del Parque Nacional Lagunas de Chacahua. Diario Oficial de la Federación.

Stuart SN, Chanson JS, Cox NA, Young BE, Rodrigues ASL, Fischman DL, et al. (2004) Status and trends of amphibians declines and extinctions worldwide. Science 306: 1783-1785.

Vilela B, Villalobos F, Rodríguez MA, Terribile LC (2014) Body size, extinction risk and knowledge bias in New World snakes. PLOS ONE 9: 1-8.

Villa-Ramírez B (1978) Especies mexicanas de vertebrados terrestres silvestres raras o en peligro de extinción. Anales del Instituto de Biología Serie Zoología 49: 303-320. 
Wilson LD, Johnson JD, Mata-Silva V (2013a) A conservation reassessment of the amphibians of Mexico based on the EVS measure. Amphibian \& Reptile Conservation 7: 97-127.

Wilson LD, Mata-Silva V, Johnson JD (2013b) A conservation reassessment of the reptiles of Mexico based on the EVS measure. Amphibian \& Reptile Conservation 7: 1-47.

Wright TF, Toft CA, Enkerlin-Hoeflich E, González-Elizondo J, Albornoz M, Rodríguez-Ferraro A, et al. (2001) Nest poaching in neotropical parrots. Conservation Biology 15: 710-720.

\section{INFORMACIÓN COMPLEMENTARIA}

https://www.academia.edu/29060205/Material_suplementario_Estado_de_conservacion_de_los_ver tebrados_terrestres_de_Oaxaca_Mexico_ERA.doc 